\title{
Mecanismos neurais da aldosterona no controle cardiovascular e do equilíbrio hidroeletrolítico
}

\author{
Central nervous system and aldosterone: Role on cardiovascular \\ control and hydro-electrolytic homeostasis
}

Silmara Formenti', Guus Hermanus Maria Schoorlemmer', Thiago Santos Moreira', ${ }^{1,2}$ Eduardo Colombari1,2

\begin{abstract}
Resumo
A aldosterona é o mais importante mineralocorticóide circulante. É secretada pela zona glomerulosa da glândula adrenal e atua em orgãos-alvo tais como os rins, cólon, glândulas salivares e sudoríparas por meio de mecanismos genômicos, a fim de manter o equilíbrio hidroeletrolítico do organismo. Mais recentemente vêm surgindo novas descobertas sobre as ações da aldosterona, as quais não se mostram restritas aos alvos clássicos de sua ação. Orgãos como coração, vasos e muitos locais do sistema nervoso central (SNC), são apontados como novos alvos da ação da aldosterona, mostrando efeitos fisiológicos bem como fisiopatológicos, que na maioria das vezes relacionam-se a mecanismos não-genômicos. Os principais efeitos da aldosterona nesses novos alvos incluem: estimulação do apetite ao sódio $\left(\mathrm{Na}^{+}\right)$, aumento da pressão arterial basal, prejuízo do barorreflexo arterial e produção de hipertrofia e fibrose no tecido cardíaco. Esses novos achados enaltecem o papel da aldosterona como o hormônio-chave no controle cardiovascular. No entanto, os mecanismos envolvidos na produção desses efeitos permanecem desconhecidos e novos estudos são necessários para o seu completo entendimento. 0 principal objetivo desta revisão será sumariar diversos estudos que indicam o envolvimento da aldosterona nos mecanismos de controle cardiovascular e do equilíbrio hidroeletrolítico do organismo.
\end{abstract}

Unitermos: Aldosterona; 11 beta-hidroxisteróide desidrogenase tipo 2 (HSD2); barorreflexo; pressão arterial; apetite ao $\mathrm{Na}^{+}$.

\section{Abstract}

Aldosterone is the most important circulating mineralocorticoid. It is secreted by the zona glomerulosa of the adrenal gland and acts on target tissues such as the kidney, colon, salivary and sweat glands by genomic mechanisms to maintain water and salt balance. More recently it has become clear that aldosterone also acts on tissues not directly involved in regulation of water and salt balance. Tissues like the heart, the vascular wall, and the central nervous system (CNS) are targets for aldosterone, which exerts its physiologic and pathophysiologic effects through both genomic and non-genomic mechanisms. The effects of aldosterone on these non-classical targets include sodium appetite, hypertension, impaired baroreceptor reflex, and cardiac hypertrophy and fibrosis. These findings reinforce the importance of aldosterone as a key cardiovascular hormone. The mechanisms that mediate these non-classical effects remain a subject of intense study. This paper reviews the involvement of aldosterone in neural mechanisms that control the cardiovascular system and water and salt balance.

Keywords: Aldosterone; 11 beta-hydroxysteroid dehydrogenase type 2 (HSD2); baroreflex; blood pressure; sodium appetite.

Recebido: 20/09/2007

Aprovado sem modificação: 02/04/2008

\footnotetext{
Departamento de Fisiologia da Universidade Federal de São Paulo - Escola Paulista de Medicina (UNIFESP-EPM), São Paulo (SP), Brasil

2 Disciplina de Fisiologia da Faculdade de Medicina do ABC (FMABC), Santo André (SP), Brasil

Endereço para correspondência: Eduardo Colombari, Disciplina de Fisiologia, Anexo I, Faculdade de Medicina do ABC, Avenida Lauro Gomes, 2000,

CEP 09060-870 - Santo André (SP), Tel.: (11) 4993-5403, Email: eduardo.colombari@pesquisador.cnpq.br
} 


\section{Introdução}

Já se passaram mais de 50 anos desde que a aldosterona foi isolada e caracterizada ${ }^{1-2}$. Desde então, sabemos que a aldosterona é o principal hormônio mineralocorticóide, sintetizado a partir do colesterol pela zona glomerulosa da glândula adrenal, que se liga a receptores mineralocorticóides (MR) específicos intra-celulares em células epiteliais-alvo. Suas ações sobre as células epiteliais são bem conhecidas e estudadas nas partes distais do néfron renal, onde atua na regulação do balanço hidroeletrolítico e subseqüente homeostase da pressão arterial, por meio do aumento na reabsorção de sódio $\left(\mathrm{Na}^{+}\right)$, água e excreção de potássio $\left(\mathrm{K}^{+}\right)$. Outros orgãos, como o cólon, as glândulas sudoríparas e salivares, também são alvos clássicos da ação mineralocorticóide da aldosterona.

Porém, como veremos nesta revisão, ao longo desses 50 anos de história da aldosterona, muito tem se descoberto sobre suas ações. Entre elas podemos destacar: 1) suas inúmeras ações em diversos orgãos, que até então não eram considerados seus alvos; 2) o provável papel fisiopatológico em tecidos não-epiteliais; 3) sua produção extra-adrenal; 4) suas ações não-genômicas; 5) a possibilidade de um receptor de membrana e 6) características necessárias para a seletividade do receptor. Novas descobertas vêm surgindo a cada dia, provando que a aldosterona é um hormônio com ações muito mais complexas do que apenas suas ações clássicas, envolvendo orgãos-alvo, com o objetivo de manter a homeostase hidroeletrolítica e a pressão arterial no nosso organismo. Hoje em dia a aldosterona é considerada um hormônio de muita importância no controle cardiovascular.

\section{0 receptor mineralocorticóide (MR) e a sua seletividade para a ação da aldosterona}

O MR, ao qual a aldosterona se liga, é classificado como um receptor nuclear, pertencente à superfamília de receptores esteróides, tireoidianos, de vitamina D e retinóides. Porém, na ausência do ligante, o MR está presente tanto no núcleo quanto no citosol, e na presença do ligante, o MR se localiza, exclusivamente, no núcleo celular ${ }^{3-4}$, onde o complexo hormônioreceptor irá modular a expressão gênica e a tradução de proteínas específicas induzidas pela aldosterona. Mas essa localização mista (núcleo/citosol) é vista apenas em MRs que não estão co-localizados com a enzima chamada $11 \beta$-hidroxiesteróide desidrogenase tipo 2 (HSD2), pois na presença de HSD2 o MR se localiza preferencialmente no citosol, assumindo a posição nuclear somente na presença do ligante ${ }^{4}$. Sugere-se que a enzima HSD2 medeie a associação entre o MR e a membrana do retículo endoplasmático na ausência do ligante, e aparentemente esse efeito é dependente de um componente estrutural da $\mathrm{HSD}_{2}{ }^{4}$, que ainda permanece desconhecido.

A enzima HSD2, dependente da coenzima nicotinamida adenina dinucleotídeo na sua forma oxidada $\left(\mathrm{NAD}^{+}\right)$, está localizada na membrana do retículo endoplasmático ${ }^{5-6}$ e sua função consiste em catalisar a conversão dos glicocorticóides endógenos, cortisol (em humanos) e corticosterona (em roedores) em seus análogos biologicamente inati- vos 11-ceto (cortisona e 11-desidrocorticosterona, respectivamente). Um estudo de hibridização in situ, mostrou que HSD2 é fortemente expressa nos rins, glândulas salivares e cólon ${ }^{7}$, orgãos considerados clássicos da ação de aldosterona.

Para a seletividade dos orgãos-alvo da aldosterona, não basta somente a presença de MR, mas deve haver a co-expressão do MR com a enzima HSD2, pois os MR são geneticamente muito semelhantes aos receptores glicocorticóides (GR) tipo $2^{8}$. A clonagem do MR mostrou uma impressionante homologia no domínio de ligação do DNA (94\%) e no domínio de ligação do ligante (54\%) com o $\mathrm{GR}^{8}$. Devido a isso, o MR é também chamado de GR tipo $1^{9-10}$. Portanto, os glicocorticóides apresentam a mesma afinidade que a aldosterona pelo $\mathrm{MR}^{11}$. Sendo assim, os MRs desprotegidos, ou seja, sem a presença de HSD2, também se deslocam para o núcleo na presença de cortisol/corticosterona ${ }^{3-4}$, sendo que o mesmo não ocorre com MRs co-localizados com HSD24. Além da alta afinidade para o MR, os glicocorticóides encontram-se em concentrações plasmáticas que são de cem a mil vezes maiores do que as concentrações plasmáticas de aldosterona $a^{4-5,12}$.

Diante da maior afinidade e da maior concentração plasmática, podemos inferir que os MRs desprotegidos são preferencialmente ocupados pelos glicocorticóides. Por isso, a presença de HSD2 nos orgãosalvo de ação da aldosterona é de grande importância, pois ela inativa os glicocorticóides, evitando que estes se liguem ao MR, permitindo acesso apenas à aldosterona.

Em um estudo de curva dose-resposta, a corticosterona in vivo mostrou uma afinidade pelo MR de apenas 3\% pelos MRs nos rins e no cólon, 30\% para os MRs no coração e 300\% pelos MRs no hipocampo $^{13}$, quando comparada à afinidade da aldosterona, mostrando o efeito protetor da enzima HSD2 sobre os MRs de tecidos que a expressam (rins e cólon).

Um exemplo para demonstrar a importância da presença da enzima HSD2 nos órgãos-alvo da aldosterona é uma doença chamada de síndrome do excesso aparente de mineralocorticóides (SEAM). Esta síndrome pode ser causada pelo consumo excessivo de substâncias que inibem a enzima HSD2 (derivados de alcaçuz - "licorice”) ${ }^{14}$ ou na sua forma mais grave, uma doença autossômica recessiva causada por uma mutação no gene que codifica a síntese de HSD2 ${ }^{15}$. Com a deficiência de HSD2, o cortisol pode livremente se ligar aos MRs, causando retenção de $\mathrm{Na}^{+}$, hipocalemia, hipertensão e supressão do sistema reninaangiotensina-aldosterona ${ }^{14-15}$, pois como demonstrado pelo trabalho de Odermatt et al. ${ }^{4}$, os MRs associados a HSD2 mutante, são deslocados para o núcleo após a ligação do cortisol.

\section{Ações genômicas x ações não-genômicas}

As ações epiteliais clássicas da aldosterona são chamadas de genômicas e são mediadas pelo receptor esteróide intracelular. Esse receptor se desloca para o núcleo da célula após a formação do complexo hormônio-receptor, modulando a expressão gênica e a tradução 
de proteínas específicas que são induzidas pela aldosterona, como por exemplo, o canal epitelial de $\mathrm{Na}^{+}$e a $\mathrm{Na}^{+}, \mathrm{K}^{+}$-ATPase. A ação genômica da aldosterona resulta de um importante período latente (de 30 a 60 minutos) prévio ao início das mudanças na atividade celular; e inibidores da transcrição (actinomicina D), da tradução (cicloheximida), da translocação do receptor esteróide e de antagonistas do MR abolem as ações genômicas. A ação genômica consiste em duas fases: precoce (de uma a seis horas) e tardia ( $>$ seis horas). A fase precoce é mediada exclusivamente pelos efeitos primários. Os efeitos primários consistem em alterações na expressão gênica que promovem ativação de canais iônicos e de proteínas de sinalização, que por sua vez, induzem as proteínas que transportam eletrólitos (efeitos secundários). A fase tardia é resultante dos efeitos primários e secundários da expressão gênica. Mais recentemente, vêm sendo especuladas as ações rápidas, não-genômicas da aldosterona, particularmente em tecidos não-epitelias tais como: células da musculatura lisa vascular, linfócitos, células endoteliais, miócitos cardíacos e células renais. Nesses tecidos foram observados os efeitos precoces causados pela aldosterona ( $<15$ minutos), que não são sensíveis aos inibidores acima citados $^{16-17}$. Acredita-se que as ações não-genômicas da aldosterona sejam mediadas por meio de outro tipo de receptor (talvez um receptor de membrana) que seja diferente do MR clássico ${ }^{18}$. Entretanto, ainda não foi possível a identificação, a caracterização e a clonagem de tal receptor ${ }^{14-15,19}$.

\section{Ações não-epiteliais}

Além das ações epiteliais da aldosterona, muitas ações não-epiteliais vêm sendo descritas ao longo dos anos, com a localização de MRs em uma variedade de tecidos não-epiteliais, particularmente no sistema cardiovascular e sistema nervoso central (SNC).

No sistema vascular, a aldosterona é conhecida por modular o tônus vascular por mecanismos ainda pouco conhecidos. Os prováveis mecanismos incluem: 1) aumento da resposta pressora às catecolaminas pela redução de sua recaptação $0^{20}$;) prejuízo da resposta vasodilatadora à acetilcolina $(\mathrm{Ach})^{21}$; 3 ) sensibilização de receptores $\beta$-adrenérgico ${ }^{22}$; 4 ) sensibilização de receptores para angiotensina II $\left.(\text { ANG II })^{23-24} ; 5\right)$ redução da biodisponibilidade do óxido nítrico $\left.{ }^{25} ; 6\right)$ efeito direto da aldosterona, possivelmente por meio de mecanismos não-genômicos. Este último mecanismo, pode ser exemplificado pelo trabalho de Romagni et al. ${ }^{26}$, que mediante a infusão intra-arterial de aldosterona, mostrou uma redução do fluxo sanguíneo. Por outro lado, estudos da literatura também mostram um aumento de fluxo sanguíneo após a infusão de aldosterona, sugerindo idéias controversas na literatura ${ }^{27-28}$. Deve-se levar em conta que essas diferenças parecem se relacionar às doses de aldosterona administradas.

No coração, a aldosterona mostra uma resposta de hipertrofia ventricular e fibrose de tecidos intersticiais e perivasculares, que são independentes de mudanças na pressão arterial sistêmica, bem como no equilíbrio hidroeletrolítico ${ }^{22-30}$. Trabalhos recentes, realizados em pacientes com insuficiência cardíaca severa, mostraram que o uso dos antagonistas de MR, espironolactona e eplerenona, em doses que não alteram significantemente a pressão arterial, reduziu a morbidade e a mortalidade desses indivíduos ${ }^{31-32}$. Em modelos animais sugere-se que eplerenona não apenas evita, como também reverte a fibrose de tecidos cardíacos ${ }^{33}$

Os mecanismos envolvidos na produção de fibrose induzida por aldosterona, não estão bem esclarecidos, mas alguns trabalhos observaram um aumento nos níveis de RNA mensageiro (RNAm), de colágeno do tipo I e III pelos fibroblastos cardíacos, em ratos com hipertensão induzida pelo modelo experimental aldosterona/sal ${ }^{34-35}$. Outro estudo, in vitro, mostrou que a aldosterona não exerce um efeito direto sobre a síntese de colágeno pelos fibroblastos cardíacos, e que provavelmente os resultados encontrados in vivo sejam resultantes de efeitos secundários à aldosterona no modelo de hipertensão induzida por mineralocorticóide ${ }^{36}$.

Inúmeros estudos mostram que a aldosterona também apresenta ações no SNC, aumentando o apetite ao $\mathrm{Na}^{+18,37-39}$ e elevando cronicamente a pressão arterial ${ }^{40-44}$.

A aldosterona não é necessária para estimular o apetite ao $\mathrm{Na}^{+}$, mas ela aumenta a ingestão de salina e potencializa os efeitos da ANG II (um hormônio peptídeo também envolvido com o apetite ao $\mathrm{Na}^{+}$). Fluharty e Epstein ${ }^{37}$ mostraram que a ação conjunta da aldosterona com a ANG II estimula o apetite ao $\mathrm{Na}^{+}$com menor latência e maior magnitude do que seria produzido com maiores quantidades de cada hormônio individualmente ${ }^{37}$. Conclusão semelhante chegaram Sakai et al..$^{38}$, que mostraram que o bloqueio de MRs centrais, mas não periféricos, com o uso do antagonista de MR, RU 28318, reduz mas não abole o apetite ao $\mathrm{Na}^{+}$induzido pelo protocolo de depleção de $\mathrm{Na}^{+}$em ratos. A eliminação da ingestão de $\mathrm{Na}^{+}$somente ocorreu após o uso concomitante de um agente que inibiu a formação de ANG II (Captopril) ${ }^{38}$. Esses resultados demonstram que o sinergismo entre a ANG II e a aldosterona é responsável pela expressão do apetite ao $\mathrm{Na}^{+}$induzido pela depleção.

Mais recentemente, Geerling et al..$^{45}$ identificaram nos núcleos do trato solitário (NTS) de ratos, a presença de neurônios sensíveis a aldosterona, por eles denominados neurônios HSD2 (proveniente de $11 \beta$-hidroxiesteróide desidrogenase do tipo 2). Esse grupamento de neurônios mostrou intensa relação com o apetite ao $\mathrm{Na}^{+} 45$ e não se correlacionou com a estimulação da sede ${ }^{46}$.

A infusão crônica intracerebroventricular (icv) de aldosterona em ratos e cachorros promove aumento da pressão arterial, entretanto, a mesma dose quando injetada sistemicamente não modifica a pressão arterial ${ }^{40-42}$.

Em ratos, a infusão icv de $5 \mathrm{ng} / \mathrm{h}$ de aldosterona elevou significantemente a pressão arterial com similar amplitude e tempo de início da resposta como quando $500 \mathrm{ng} / \mathrm{h}$ são injetados subcutâneamente ${ }^{40}$. A resposta foi dose-dependente e a sensibilização pela redução da massa renal (nefrectomia unilateral) e a carga de sal acentuaram o desenvolvimento da hipertensão, mas não foram essenciais ${ }^{40,47-48}$. 
A resposta pressora promovida pela infusão icv de aldosterona foi bloqueada pela administração prévia do antagonista de MR, prorenone, em doses que não possuem efeitos quando injetadas sistemicamente ${ }^{40}$. A infusão icv de outro antagonista de MR, RU 28318, também bloqueou a hipertensão produzida pela infusão subcutânea (sc) de aldosterona, o que não ocorreu com a mesma dose do antagonista injetado subcutaneamente ${ }^{43}$. Do mesmo modo, infusão icv de corticosterona bloqueou, de maneira dose-dependente, os efeitos pressóricos da aldosterona ${ }^{44}$. A injeção icv de corticosterona não exerceu nenhum efeito sobre a pressão $\operatorname{arterial}^{44}$.

Nos modelos de ratos com hipertensão induzida pela administração do agonista mineralocorticóide acetato de desoxicorticosterona associada à ingestão de salina (DOCA/sal), os componentes simpático e parassimpático do barorreflexo se encontram atenuados nos estágios pré-hipertensivos ${ }^{49-52}$. Neste modelo de hipertensão, a injeção icv crônica do antagonista de MR, RU 28318, significantemente atenuou a hipertensão, e essa redução foi atribuída a mecanismos que envolveram a restauração do barorreflexo e uma redução do tônus vasomotor simpático ${ }^{53}$. Um trabalho que também usou o modelo crônico de hipertensão $\mathrm{DOCA} / \mathrm{sal}$ (três e cinco semanas) ${ }^{54}$ mostrou que uma única injeção icv de RU28318 em ratos tratados com DOCA/sal por três semanas (período no qual a hipertensão não está totalmente estabelecida), reduziu a pressão arterial sistólica após duas horas, medida por método indireto (pressão de cauda). Porém, a medida direta da pressão arterial (canulação) mostrou um aumento da pressão arterial média (PAM) após a injeção única icv de RU 28318, que perdurou durante todo o ciclo claro $(\sim 10 \mathrm{~h})$, havendo importante queda da PAM no ciclo escuro $(\sim 17 \mathrm{~h}$ após a injeção icv). Nos ratos tratados com DOCA/sal por cinco semanas, o aumento de pressão arterial já está estabelecido, a injeção única icv de RU 28318 não afetou a pressão arterial basal, mostrando que o bloqueio do MR não afeta a pressão arterial basal após o estabelecimento da hipertensão ${ }^{53}$. Esses experimentos mostram que a integridade funcional do MR é relevante para o desenvolvimento da hipertensão mineralocorticóide ${ }^{53-54}$

O bloqueio do MR com o antagonista RU 28318 também foi capaz de inibir o desenvolvimento da hipertensão no modelo de ratos Dahl S/JR (sensíveis a sal) e também de reduzir os níveis hipertensivos após duas semanas de dieta hipersódica, quando a hipertensão já está estabelecida ${ }^{55}$.

Todos esses resultados evidenciam o envolvimento dos MRs centrais no desenvolvimento da hipertensão induzida por mineralocorticóides. As doses infundidas foram muitas ordens de magnitude inferiores às quantidades necessárias para a resposta sistêmica, sugerindo assim, função primária do SNC para o efeito hipertensivo dos mineralocorticóides. O bloqueio da hipertensão com o uso de antagonistas seletivos de MR sugere que a resposta pressórica seja mediada pelo MR clássico, e o antagonismo causado pela corticosterona, sugere que o MR envolvido seja desprotegido da ação da HSD2. No entanto, a exata localização anatômica das regiões en- cefálicas envolvidas na resposta pressórica dos mineralocorticóides precisa ainda ser mais bem elucidada. Regiões hipotalâmicas periventriculares são os locais sugeridos como o possível local de ação da aldosterona para aumento da pressão arterial ${ }^{56-57}$. No entanto, como a aldosterona e os antagonistas de MR foram injetados no ventrículo lateral e a resposta pressora apareceu após dez a 14 dias $^{40-41}$, pode ter ocorrido difusão dessas substâncias para outros locais do SNC.

\section{Efeitos da aldosterona no mecanismo do barorreflexo}

Estudos em humanos e em ratos demonstram uma importante relação entre a aldosterona e a atenuação da resposta do barorreflexo. Os trabalhos de um mesmo laboratório ${ }^{50,52}$ sugerem que ratos tratados no modelo DOCA/sal apresentam uma redução nas respostas de atuação do barorreflexo via estimulação do nervo depressor aórtico ${ }^{50}$ e injeção intravenosa (iv) de noradrenalina ${ }^{52}$, ainda nos estágios préhipertensivos. Esses experimentos sugerem que o prejuízo do barorreflexo pode contribuir no desenvolvimento da hipertensão em ratos tratados com DOCA/sal.

Em cachorros, tanto a infusão aguda, quanto à infusão crônica de aldosterona, foram capazes de reduzir a atividade dos barorreceptores ${ }^{20,58}$. A infusão prévia do antagonista de MR, espironolactona, foi capaz de impedir a redução da atividade dos barorreceptores promovida pela aldosterona, sugerindo a participação dos MRs na atividade do barorreflexo ${ }^{20}$. Curiosamente essa resposta também foi bloqueada pela remoção das células endoteliais do seio carotídeo ${ }^{20}$, o que sugere que a aldosterona pode estimular as células endoteliais a liberarem uma substância desconhecida que diminui a atividade dos barorreceptores. A infusão crônica de aldosterona também foi capaz de reduzir a atividade do barorreflexo. Essa redução também foi bloqueada com a infusão prévia de espironolactona ${ }^{58}$. No mesmo estudo, os autores mostraram que a inibição da $\mathrm{Na}^{+}, \mathrm{K}^{+}$- ATPase, pela infusão de ouabaína, foi capaz de atenuar os efeitos da inibição do barorreflexo pela aldosterona ${ }^{58}$. Isso sugere uma participação da $\mathrm{Na}^{+}, \mathrm{K}^{+}$- ATPase nessas respostas, uma vez que a aldosterona estimula a atividade da $\mathrm{Na}^{+}, \mathrm{K}^{+}-$ATPase $^{59-60}$

A infusão central crônica de aldosterona, também foi avaliada em ratos Dahl-sensíveis e -resistentes a sal ${ }^{61}$. Observou-se que, nos ratos sensíveis ao sal, a infusão icv de aldosterona causou um aumento da atividade simpática, hipertensão e prejuízo no controle do barorreflexo sobre a atividade simpática renal e sobre a frequiência cardíaca $^{61}$. Resultados semelhantes foram observados em humanos. Yee e Struthers ${ }^{62}$ realizaram um estudo em voluntários saudáveis e normotensos, mostrando que a infusão aguda iv de aldosterona prejudicou o controle do barorreflexo sobre a freqüência cardíaca, com acentuada diminuição da resposta de bradicardia a estimulação do barorreceptor arterial elicitado pela infusão iv de fenilefrina. Esses efeitos foram atribuídos como um prejuízo do componente paras- 
simpático do barorreflexo, sem alterações no componente simpático cardíaco, uma vez que, a resposta de freqüência cardíaca à infusão de nitroprussiato de sódio não se mostrou alterada ${ }^{62}$.

Heindl et al. ${ }^{63}$ observaram que a infusão (iv) de aldosterona resultou em uma tendência em aumentar a atividade cardíaca vagal e aumentar a resposta de taquicardia ao nitroprussiato de sódio, sem afetar o controle do barorreflexo sobre a atividade simpática para o leito muscular esquelético. Resultados diferentes foram encontrados por Monahan et al. ${ }^{64}$ que observaram um prejuízo de ambos os componentes do barorreflexo, parassimpático e simpático, após infusão iv de aldosterona.

Os diferentes resultados encontrados nesses estudos em humanos possivelmente se relacionaram com as diferentes doses utilizadas, diferentes técnicas utilizadas para estimular o barorreflexo, ou também, pelo aumento da concentração plasmática de aldosterona bem acima dos valores fisiológicos ${ }^{62-64}$. Uma elevação nos níveis plasmáticos de aldosterona pode ser necessária para atenuar o barorreflexo, o que se aproximaria de situações patológicas que envolvem o excesso de aldosterona, como por exemplo, a hipertensão dependente de aldosterona e em situações de insuficiência cardíaca. No entanto, os mecanismos pelos quais a aldosterona promove esses efeitos sobre o barorreflexo ainda não estão esclarecidos.

As diferentes respostas encontradas em relação à alteração no componente simpático do barorreflexo, evidenciada pela atividade simpática muscular esquelética, necessita de mais estudos, usando modelos animais, a fim de investigar o possível prejuízo da modulação do tônus vasoconstritor simpático para diferentes leitos.

Apesar das diferenças entre as diversas respostas encontradas nos estudos com animais e humanos, é inegável que a aldosterona está envolvida de alguma forma no prejuízo da função do barorreflexo, porém os mecanismos envolvidos ainda permanecem desconhecidos e necessitam de novas investigações.

\section{Neurônios sensíveis à aldosterona no NTS: os neurônios HSD2}

Além de ser expressa nos orgãos clássicos de ação da aldosterona ${ }^{7}$, um estudo de hibridização in situ ${ }^{65}$ mostrou a presença da expressão de RNAm para HSD2 no SNC. Importante expressão de HSD2 foi encontrada na porção comissural do NTS e no órgão subcomissural (SCO, um órgão circunventricular localizado abaixo da comissura posterior). Ademais, níveis moderados de expressão foram vistos na porção ventrolateral do hipotálamo ventromedial (VMHvl) e algumas células no núcleo vestibular medial (MV) encontraram-se marcadas para RNAm de $\mathrm{HSD}_{2}{ }^{65}$. Imunorreatividade para $\mathrm{MR}^{66}$ e para RNAm de $\mathrm{MR}^{67}$ já foram descritas na porção medial do NTS. O NTS medial e comissural estão diretamente envolvidos no controle cardiovascular, pois lesões que acometem simultaneamente essas duas áreas resultam em hipertensão aguda fulminante ${ }^{68-69}$.
A partir dessas informações, Geerling et al. ${ }^{45}$ realizaram estudos com o objetivo de identificar locais no SNC que pudessem ser sensíveis à aldosterona. Utilizando um anticorpo específico contra MR de rato, observaram uma expressão difusa de MR por todo o encefálo, corroborando com estudos prévios ${ }^{66,70}$. Porém, como já descrito anteriormente, apenas a presença de MR não é suficiente para que o tecido seja seletivo à aldosterona. Diante disso, foi avaliado se estes neurônios contendo MR também expressavam a enzima HSD2, usando um anticorpo contra a seqüência peptídica única de HSD2 do rato ${ }^{45}$. Com os tratamentos anatômicos específicos descobriu-se uma intensa imunorreatividade no NTS medial, com a mesma distribuição restrita apresentada pelos MR no NTS. Com a dupla marcação observaram que a enzima HSD2 estava co-localizada com os MR nucleares e perinucleares. Sendo assim, esses neurônios receberam a denominação de "neurônios HSD2", por serem neurônios que expressam a enzima $11 \beta$-hidroxiesteróide desidrogenase do tipo $2^{45}$.

Os neurônios HSD2 possuem localização rostral à área postrema (AP), se estendendo até a porção caudal do NTS ao nível da transição bulbo-espinal. As concentrações mais densas de neurônios HSD2 localizam-se entre o NTS medial que está na borda do quarto ventrículo, próximo ao nível que se forma o canal central, abaixo da $\mathrm{AP}^{39,45}$. Assim, como Roland et al. ${ }^{65}$, Geerling et al. ${ }^{45}$ também encontraram, ao examinar todo o encéfalo, imunorreatividade para HSD2 no SCO, no VMHvl e em poucas células no MV rostral ao NTS, porém nenhum desses locais apresentou co-localização de MR e HSD2. Sendo assim, os neurônios HSD2 do NTS são considerados os únicos neurônios sensíveis à aldosterona no $\mathrm{SNC}^{45}$.

Além da co-localização de MR e HSD2 no NTS medial, os dendritos dos neurônios HSD2 se estendem para a região vascular do NTS abaixo da $\mathrm{AP}^{45}$. A AP consiste em um orgão circunventricular desprovido de barreira hemato-encefálica (BHE) ${ }^{71}$, o que é outra característica importante para ação da aldosterona no SNC, uma vez que, relativamente baixos níveis de aldosterona conseguem atravessar a BHE ${ }^{13,72}$. Essa informação poderia ser irrelevante se realmente se comprovasse a produção local de aldosterona pelo SNC, porém até o momento, apenas encontraram a expressão no SNC de enzimas envolvidas na síntese de aldosterona ${ }^{12,73-76}$, o que não comprova a produção local de aldosterona no SNC. Portanto, devido a localização próxima à AP, os neurônios HSD2 no NTS estão em uma área de fácil acesso para a aldosterona circulante.

O NTS dorso-medial constitui o principal sítio de entrada das aferências dos barorreceptores e em menor escala, também, recebe aferências dos quimiorreceptores e aferências gastrointestinais ${ }^{77-79}$, portanto, os neurônios HSD2 podem ter um papel na integração de sinais neurais e humorais dos estados cardiovasculares ou gastrointestinais ${ }^{45}$. De posse de todas essas informações, Geerling et al. ${ }^{39} \mathrm{pu}-$ deram comprovar que os neurônios HSD2 estão intimamente relacionados ao controle do apetite ao $\mathrm{Na}^{+}$. Primeiro, foi mostrado que os neurônios HSD2 são sensíveis e seletivos à aldosterona, por meio 
da análise, in vivo, da translocação nuclear dos MR em ratos adrenalectomizados ( $\mathrm{adx}$ ) que receberam infusão de aldosterona, veículo ( $1 \%$ de etanol em $0.9 \%$ de salina) e corticosterona. Os ratos infundidos com aldosterona mostraram a maioria dos neurônios HSD2 com densa imunorreatividade nuclear $\left(62,7 \pm 4,7 \% ; \mathrm{p}<10^{-7}\right.$, comparado ao veículo). Em contraste, a corticosterona teve apenas um pequeno efeito sobre a translocação nuclear dos MRs dos neurônios HSD2 $(19,4 \pm 4,5 \%$; $\mathrm{p}<0,001$, comparado ao grupo aldosterona), apesar dos níveis de concentração cem vezes maiores ${ }^{39}$, mostrando o efeito protetor da enzima HSD2 ao MR. No mesmo trabalho, os autores mostraram que os neurônios HSD2 são ativados por diversas situações que estimulam o apetite ao $\mathrm{Na}^{+}$(dieta de privação de $\mathrm{Na}^{+}$, diurese por furosemida, tratamento crônico com altas doses de DOCA e adrenalectomia). A maior ativação foi observada no protocolo de oito dias de privação de $\mathrm{Na}^{+}$sem acesso a salina (32,5 $\pm 4,5 \%$ de expressão de proteína c-Fos pelos neurônios HSD2), comparado à diurese por furosemida associada a privação de $\mathrm{Na}^{+}$a curto prazo (24 - 48 horas; $18,7 \pm 6,8 \%$ e $29,4 \pm 4,7 \%$, respectivamente) e DOCA sem privação de $\mathrm{Na}^{+}$(com acesso a 3\% NaCl; 20,9 $\pm 2,5 \%$; $<<0,001$ vs veículo). Essa ativação se relacionou a uma importante e rápida ingesta de $\mathrm{Na}^{+}$ mostrada no trabalho pelos grupos de oito dias de privação de $\mathrm{Na}^{+} \mathrm{e}$ DOCA sem privação de $\mathrm{Na}^{+39}$. Outro resultado interessante foi a rápida inativação dos neurônios HSD2 após acesso a salina hipertônica. Ratos que foram privados de $\mathrm{Na}^{+}$por oito dias apresentaram uma rápida inativação dos neurônios HSD2, sendo praticamente abolida após duas horas de acesso a 3\% $\mathrm{NaCl}(4,7 \pm 0,6 \%$ de c-Fos nuclear; $\mathrm{p}=0,0001$, vs oito dias de privação de $\mathrm{Na}^{+}$sem acesso a salina). Durante essas duas horas de acesso a salina, esses ratos beberam 7,5 $\pm 0,6 \mathrm{~mL}$ de $3 \%$ de $\mathrm{NaCl}\left(\mathrm{p}<10^{-7}\right.$ vs a média antes da privação, 1,67 $\pm 0,4 \mathrm{~mL})$, primariamente nos primeiros 30 minutos $(6,7 \pm 0,7 \mathrm{~mL})^{39}$. Curiosamente, os ratos adx e privados de $\mathrm{Na}^{+}$(um a seis dias), apresentaram um grande aumento de expressão de c-Fos pelos neurônios HSD2 no NTS após um dia de privação de $\mathrm{Na}^{+}(36,6 \pm 3,3 \%)$ e que permaneceu elevada pelos seis dias restantes. Um grupo adicional de ratos adx privados de $\mathrm{Na}^{+}$por três dias, bebeu voluntariamente uma grande quantidade de salina hipertônica $(28,5 \pm 0,8 \mathrm{~mL}$, além de 24 horas), confirmando o seu robusto apetite ao $\mathrm{Na}^{+}$. Vale lembrar que a ingestão de salina hipertônica em 24 horas também inibiu a ativação dos neurônios HSD2 neste modelo $(2,9 \pm 1,4 \%$; p = 0,01, vs 3 dias de privação sem acesso a salina). Esse resultado mostrou que, além de serem ativados pela aldosterona, os neurônios HSD2 também são ativados em situações de depleção de $\mathrm{Na}^{+}$, por outro mecanismo (neural/humoral) independente da aldosterona ${ }^{39}$, que ainda permanece desconhecido.

O trabalho de Wang e Edwards ${ }^{80}$ avaliou os diferentes efeitos do tamanho da lesão no bulbo dorsomedial sobre os comportamentos ingestivos de ratos, sugerindo que a porção do NTS ao redor da AP teria uma importante contribuição para os efeitos comportamentais observados após lesões na AP. Com relação à ingesta de $\mathrm{Na}^{+}$, os autores observaram que animais depletados de $\mathrm{Na}^{+}$com lesões menores do NTS adjacente a $\mathrm{AP}$, ingeriam mais $\mathrm{Na}^{+}$do que os animais com lesões maiores ${ }^{80}$, o que nos leva a pensar que nos animais com lesões envolvendo uma maior parte do NTS adjacente a AP, os neurônios HSD2 também podem ter sido lesados e por isso a ingestão de $\mathrm{Na}^{+}$foi reduzida em comparação a lesão menor.

Outro resultado interessante foi encontrado durante o tratamento mineralocorticóide crônico, sem acesso a salina ${ }^{81}$. Como já observado, os neurônios HSD2 são ativados em paralelo ao apetite ao $\mathrm{Na}^{+}$por depleção de $\mathrm{Na}^{+}$ou por tratamento mineralocorticóide crônico na ausência de depleção de $\mathrm{Na}^{+}{ }^{39}$. Porém, após o tratamento mineralocorticóide crônico (2 mg/dia de DOCA, sete dias), os ratos tiveram acesso a salina hipertônica $(3 \% \mathrm{NaCl})$ por duas horas, e beberam oito vezes mais salina do que o grupo controle $(0,4 \pm 0,1 \mathrm{~mL}$ veículo $+\mathrm{NaCl}$ vs 3,5 0,7 $\mathrm{mL}$ DOCA $+\mathrm{NaCl}$ ), observando-se que o maior consumo ocorreu nos primeiros 30 minutos, $3,3 \pm 0,8 \mathrm{~mL}$, de um total ingerido em duas horas de 3,5 $\pm 0,7 \mathrm{~mL}^{81}$. Mesmo mantendo o tratamento mineralocorticóide, os neurônios HSD2 no NTS foram inibidos (aproximadamente 50\% de redução da ativação) pela ingestão de salina hipertônica. Esses achados sugerem que os neurônios HSD2 são inibidos por sinais diretamente relacionados à ingestão de salina, e não pelo simples alívio da deficiência de $\mathrm{Na}^{+}$, que não ocorre durante a administração de mineralocorticóides ${ }^{81}$.

Como o NTS integra muitas informações viscerosensoriais, incluíndo as aferências de receptores gastrointestinais, osmorreceptores hepáticos, aferências gustatórias e de receptores cardiovasculares ${ }^{78-79}$, os neurônios HSD2 poderiam ser inibidos por mecanismos inibitórios locais $^{82}$, uma vez que, como observado após a ingestão de salina hipertônica os neurônios HSD2 foram inativados e os neurônios vizinhos a eles no NTS medial foram concomitantemente ativados ${ }^{81}$.

Como evidenciado por esses trabalhos, os neurônios HSD2 são neurônios-alvo da ação de aldosterona no $\mathrm{SNC}^{45}$ e são ativados em paralelo com o apetite ao $\mathrm{Na}^{+}$durante o tratamento com altas doses do agonista mineralocorticóide (DOCA), bem como pela dieta hipossódica, que aumenta a produção de aldosterona ${ }^{39}$. Além disso, os neurônios HSD2 recebem informações sobre a depleção de $\mathrm{Na}^{+}$por meio de outras fontes além da aldosterona ${ }^{39}$, e são diretamente inibidos por sinais relacionados à ingestão de salina hipertônica ${ }^{81}$, sem que estejam relacionados à estimulação da sede ${ }^{46}$. Todas essas informações caracterizam os neurônios HSD2 como parte integrante de um circuito neuro-humoral relacionado ao controle do apetite ao $\mathrm{Na}^{+}$.

Estudos com uso de traçadores neuronais mostraram importantes projeções eferentes dos neurônios HSD2 do NTS para a porção ventrolateral do núcleo leito da estria terminal (BSTvl), pré-locus ceruleus (pre-LC) e para divisão interna do núcleo parabraquial externo-lateral (PBel). Sendo que a BSTvl recebe projeções eferentes dos neurônios HSD2 tanto direta, quanto indiretamente por meio do pre-LC e do $\mathrm{PBel}^{83}$. Não foram encontradas conexões entre os neurônios HSD2 e locais envolvidos no controle autonômico ${ }^{83}$, sugerindo que os neurônios HSD2 provavelmente não modulem o sistema nervoso autônomo diretamente por projeções do NTS 
para regiões ventrolaterais do bulbo. No entanto, não podemos descartar o possível envolvimento dos neurônios HSD2 sobre a integração que acontece no NTS dos ajustes cardiovasculares modulados no próprio NTS.

Outro estudo demonstrou que os neurônios HSD2 apresentam conexões bidirecionais com o núcleo central da amígdala $(\mathrm{CeA})^{84}$, região essa que está relacionada com a modulação do apetite ao $\mathrm{Na}^{+}$, em resposta a administração de mineralocorticóides ${ }^{18,85}$. Geerling e Loewy ${ }^{84}$ sugerem que os neurônios HSD2 enviam projeções indiretas, via PBel, para a porção lateral do CeA (CeA-L) e que a porção medial do $\mathrm{CeA}(\mathrm{mCeA})$ enviaria uma projeção descendente direta aos neurônios HSD2, formando assim um importante circuito para modulação do apetite ao $\mathrm{Na}^{+}$, no qual o aumento deste apetite em resposta à administração de mineralocorticóides ao CeA, seria ao menos em parte, devido a um impulso indireto dos neurônios HSD2, que poderiam fornecer informações sobre a necessidade de $\mathrm{Na}^{+}$no organismo ${ }^{84}$.

Os neurônios HSD2 também se projetam para o núcleo accumbens (NAc), por meio de uma via multisináptica ${ }^{86}$. O NAc está envolvido nos mecanismos de recompensa, motivação e aprendizado, e se torna ativado durante vários comportamentos ingestivos, incluindo a ingestão de $\mathrm{Na}^{+87}$. A via multisináptica proposta por Shekhtman et al. ${ }^{86}$ envolveria os neurônios HSD2 que se projetam para o PBel e pre-LC, essas estruturas pontinas se projetam para o núcleo paraventricular do hipotálamo (PVH), que então enviaria as informações provenientes dos neurônios HSD2 para o NAc. Esta via pode ter um papel na motivação do comportamento e procura por sal em ratos depletados de $\mathrm{Na}^{+86}$.

Além dessas conexões com várias estruturas encefálicas, os neurônios HSD2 também recebem informações de microcircuitos locais que envolvem a AP e os neurônios neurotensinérgicos ${ }^{82}$. A AP é um órgão circunventricular que diretamente inerva o $\mathrm{NTS}^{71}$ e exerce um importante efeito inibitório sobre o apetite ao $\mathrm{Na}^{+80,88-90}$. Mesmo com lesões de AP os animais mantêm o apetite ao $\mathrm{Na}^{+}$em resposta à depleção de $\mathrm{Na}^{+}$, sugerindo que o circuito neural responsável pelo controle do apetite ao $\mathrm{Na}^{+}$esteja preservado ${ }^{91}$. Como já citado anteriormente, lesões de AP associada a lesões do NTS adjacente, mostram diferentes respostas à ingestão de $\mathrm{Na}^{+}$relacionadas ao tamanho da lesão no NTS adjacente ${ }^{80}$. Isso pode sugerir que os neurônios da AP sensíveis ao $\mathrm{Na}^{+}$ poderiam inervar os neurônios HSD2 $2^{82}$.

No estudo de Sequeira et al. ${ }^{82}$, observou-se que os axônios dos neurônios da AP que se projetam ao NTS estão em justaposição aos neurônios HSD2 e aos neurônios neurotensinérgicos, e os botões neurotensinérgicos estão em justaposição aos neurônios HSD2, sugerindo que este microcircuito possa ser o local de integração para informações aferentes sobre as condições cardiovascular e de $\mathrm{Na}^{+}$plasmático.
Apesar dos neurônios HSD2 não mostrarem nenhuma conexão direta com o sistema nervoso autônomo ${ }^{83}$, não se pode excluir que eles possam influenciá-lo de maneira indireta, uma vez que, como vimos, os neurônios HSD2 se localizam no NTS, um local do SNC de importante integração de informações viscerossensoriais ${ }^{77-79}$ e mais precisamente se localizam no NTS medial ${ }^{45}$, importante área de controle cardiovascular $^{77-79}$. Além disso, o NTS também participa de vias multissinápticas envolvendo várias estruturas que de alguma forma, seja direta ou indiretamente, se relacionam aos neurônios HSD2.

Os neurônios HSD2 são sensíveis e seletivos à aldosterona e também são ativados pela infusão de DOCA ${ }^{39}$. Como já discutido anteriormente, a aldosterona além de estimular o apetite ao $\mathrm{Na}^{+18,37-39}$, também está relacionada com o aumento da pressão arterial, por atuar em seus MRs centrais ${ }^{40-44}$, apesar de sua exata localização não estar bem esclarecida. Porém, o MR envolvido na hipertensão parece ser desprotegido, pois permite que a corticosterona de maneira dose-dependente, antagonize o efeito hipertensivo da aldosterona ${ }^{44}$, o que não exclui a possibilidade de que haja um escape de corticosterona à ação de $\mathrm{HSD} 2^{13}$, por exemplo, por saturação da enzima ${ }^{4}$.

A aldosterona está relacionada ao prejuízo do barorreflexo tanto em humanos ${ }^{62-64}$ quanto em animais ${ }^{20,49-52,58,61}$, por mecanismos ainda desconhecidos. Em modelos animais de hipertensão mineralocorticóide o barorreflexo já se encontra atenuado em estágios pré-hipertensivos ${ }^{49-}$ 53. Animais Dahl-sensíveis a sal, que mostram uma regulação anormal do barorreflexo antes mesmo do desenvolvimento de hipertensão, apresentam 20\% a mais de neurônios HSD2 no NTS quando comparados aos ratos Dahl-resistentes à sal, Sprague-Dawley, Wistar Kyoto e espontaneamente hipertensos $(\mathrm{SHR})^{92}$.

Em suma, muito se descobriu sobre a aldosterona e suas ações nesses últimos anos. Esses achados vêm contrariando o dogma de que a aldosterona atua excluvisamente em alvos epiteliais por meio de um mecanismo genômico para modular o balanço hidroeletrolítico. No entanto, está claro que a aldosterona tem importantes efeitos fisiológicos e fisiopatológicos sobre alvos não-epiteliais, dos quais muitos parecem ocorrer por mecanismos não-genômicos. E os recentes avanços aumentam o número de questões sobre de que forma a aldosterona atua para estimular o apetite ao $\mathrm{Na}^{+}$, causar hipertensão arterial por meio de mecanismos neurais, interferir no mecanismo do barorreflexo e causar hipertrofia e fibrose no coração. As respostas para essas questões ainda permanecem desconhecidas e necessitam de novas pesquisas, pois provavelmente levarão à descoberta de novos e melhores tratamentos para doenças como hipertensão arterial e insuficiência cardíaca. No entanto, muitos estudos ainda são necessários para se encontrar essas respostas, e provavelmente os próximos 50 anos de história da aldosterona serão no mínimo muito interessantes. 


\section{Referências}

1. Simpson AS, Tait JF, Wettstein A, Neher R, von Euw J, Reichstein T. Isolation from the adrenals of a new crystalline hormone with specially high effectiveness on mineral metabolism. Experientia 1953:9:333-5.

2. Tait SA, Tait JF, Coghlan JP. The discovery, isolation and identification of aldosterone: reflections on emerging regulation and function. Mol Cell Endocrinol 2004;217(1-2):1-21.

3. Fejes-Tóth G, Pearce D, Náray-Feje-Tóth A. Subcellular localization of mineralocorticoid receptors in living cells: Effects of receptor agonists and antagonists. Proc Natl Acad Sci USA 1998;95(6):2973-8.

4. Odermatt A, Arnold P, Frey FJ. The intracellular localization of the mineralocorticoid receptor is regulated by 11 beta-hydroxysteroid dehydrogenase type 2. J Biol Chem 2001;276(30):28484-92

5. Náray-Fejes-Tóth A, Fejes-Tóth G. Subcellular localization of the type 211 beta- hydroxysteroid dehydrogenase. J Biol Chem 1996;271(26):15436-42.

6. Odermatt A, Arnold P, Stauffer A, Frey BM, Frey FJ. The N-terminal anchor sequences of 11 beta-hydroxysteroid dehydrogenase determine their orientation in the endoplasmatic reticulum membrane. J Biol Chem 1999;274(40):28762-70.

7. Roland BL, Funder JW. Localization of 11 beta-hydroxysteroid dehydrogenase type 2 in rat tissues: in situ studies. Endocrinology 1996;137(3):1123-8

8. Arriza JL, Weinberger C, Cerelli G, Glaser TM, Handelin BL, Housman $D E$, et al. Cloning of human mineralocorticoid receptor complementary DNA: structural and functional kinship with glucorticoid receptor. Science 1987:237(4812):268-75

9. Reul JM, de Kloet ER. Two receptor systems for corticosterone in rat brain: microdistribution and differential occupation. Endocrinology 1985;117(6):2505-11.

10. Ito T, Morita N, Nishi M, Kawata M. In vitro and in vivo immunocytochemistry for distribution of mineralocorticoid receptor with the use of specific antibody. Neurosci Res 2000;37(3):173-82.

11. Shepard KE, Funder JW. Equivalent affinity of aldosterone and corticosterone for type I receptors in kidney and hippocampus: direct binding studies. $J$ Steroid Biochem 1987;28(6):737-42.

12. Gomez-Sanchez EP, Gomez-Sanchez CE. Is aldosterone synthesized in the CNS regulated and functional? Trends Endocrinol Metab 2003;14(10):444-6.

13. Funder J, Myles K. Exclusion of corticosterone from epithelial mineralocorticoid receptors is insufficient for selectivity of aldosterone action: in vivo binding studies. Endocrinology 1996;137(12):5264-8.

14. Edwards CR, Walker BR, Benediktisson R, Seckl JR. Congenital and acquired syndromes of apparent mineralocorticoid excess. J Steroid Biochem Mol Biol 1993;45(1-3):1-5

15. Stewart PM, Krozowski ZS, Gupta A, Milford DV, Howie AJ, Sheppard $\mathrm{MC}$, et al. Hypertension in the syndrome of apparent mineralocorticoid excess due to mutation of the 11 beta-hydroxysteroid dehydrogenase type 2 gene. Lancet 1996;347(8994):88-91.

16. Booth RE, Johnson JP, Stockand JD. Aldosterone. Adv Physiol Educ 2002;26(1-4):8-20.

17. Connell JMC, Davies E. The new biology of aldosterone. J Endocrinol 2005;186(1):1-20

18. Sakai RR, McEwen BS, Fluharty SJ, Ma LY. The amygdala: Site of genomic and nongenomic arousal of aldosterone-induced sodium intake. Kidney Int 2000;57(4):1337-45

19. Falkenstein E, Christ M, Feuring M, Wehling M. Specific nongenomic actions of aldosterone. Kidney Int 2000;57(4):1390-4.

20. Wang W, McClain JM, Zucker $\mathbb{H}$. Aldosterone reduces baroreceptor discharge in the dog. Hypertension 1992;19(3):270-77.

21. Taddei S, Virdis A, Mattei P, Salvetti A. Vasodilation to acetylcholine in primary and secondary forms of human hypertension. Hypertension 1993;21 (6 pt 2):929-33.

22. Jazayeri A, Meyer WJ. Mineralocorticoid-induced increased in betaadrenergic receptors of cultured rat arterial smooth muscle cells. J Steroid Biochem 1989;33(5):987-91.

23. Schiffrin EL, Gutkowska J, Genest J. Effect of angiotensin II and deoxycorticosterone infusion on vascular angiotensin II receptor in rats. Am J Physiol 1984;246(4 pt 2):H608- H614.

24. Schiffrin EL, Franks DJ, Gutkowska J. Effect of aldosterone on vascular angiotensin II receptors in the rat. Can J Physiol Pharmacol 1985:63(12):1522-7.

25. Chun TY, Bloem LJ, Pratt JH. Aldosterone inhibits inducible nitric oxide synthase in neonatal rat cardiomyocytes. Endocrinology 2003;144(5):1712-7.

26. Romagni P, Rossi F, Guerrini L, Quirini C, Santiemma V. Aldosterone induces contraction of the resistance arteries in man. Atherosclerosis 2003;166(20:345-9.

27. Schmitd BM, Oehmer S, Delles C, Bratke R, Schneider MP, Klingbeil A, et al. Rapid nongenomic effects of aldosterona on human forearm vasculature. Hypertension 2003;42(2):156-60.

28. Uhrenholt TR, Schjerning J, Hansen PB, Norregaard R, Jensen BL, Sorensen $\mathrm{GL}$, et al. Rapid inhibition of vasoconstriction in renal afferent arterioles by aldosterone. Circ Res 2003;93(12):1258-66.

29. Brilla CG, Weber KT. Mineralocorticoid excess, dietary sodium, and myocardial fibrosis. J Lab Clin Med 1992;120(6):893-901.

30. Rocha R, Stier CT Jr. Pathophysiological effects of aldosterone in cardiovascular tissues. Trends Endocr Metab 2001:12(7):308-14.

31. Pitt B, Zannad F, Remme WJ, Cody R, Castaigne A, Perez A, et al. The effect of spironolactone on morbidity and mortality in patients with severe heart failure. Randomized Aldactone Evaluation Study Investigators. N Engl J Med 1999;341(10):709-17. 
32. Pitt B, Remme WJ, Zannad F, Neaton J, Martinez F, Roniker B, et al. Eplerenone, a selective aldosterona blocker, in patients with left ventricular dysfunction after myocardial infarction. New Engl $\mathrm{J}$ Med 2003;348(14):1309-21.

33. Young M, Funder JW. Eplerenone, but not steroid withdrawl, reverses cardiac fibrosis in deoxycorticosterone/salt-treated rats. Endocrinology 2004;145(7):3153-7.

34. Robert V, Van Thiem N, Cheav SL, Mouas C, Swynghedauw B, Delcayre C. Increased cardiac types I and III collagen mRNAs in aldosterone-salt hypertension. Hypertension 1994;24(1):30-6.

35. Robert V, Silvestre JS, Charlemagne D, Sabri A, Trouvé P, Wassef M, et al. Biological determinants of aldosterone-induced cardiac fibrosis in rats. Hypertension 1995;26(6 pt 1):971-8.

36. Fullerton MJ, Funder JW. Aldosterone and cardiac fibrosis: in vitro studies. Cardiovasc Res 1994;28(12):1863-7.

37. Fluharty SJ, Epstein AN. Sodium appetite elicited by intracerebroventricular infusion of angiotensina II in the rat: II. Synergistic interaction with systemic mineralocorticoids. Behav Nerosci 1983;97(5):746-58.

38. Sakai RR, Nicolaidis S, Epstein AN. Salt appetite is supressed by interference with angiotensin II and aldosterone. Am J Physiol 1986;251 (4 pt 2):R762-8.

39. Geerling JC, Engeland WC, Kawata M, Loewy AD. Aldosterone target neurons in the nucleus tractus solitarius drive sodium appetite. J Neurosci 2006;26(2):411-7

40. Gomez-Sanchez EP. Intracerebroventricular infusion of aldosterone induces hypertension in rats. Endocrinology 1986;118(2):819-23.

41. Kageyama Y, Bravo EL. Hypertensive mechanisms associated with centrally administered aldosterone in dogs. Hypertension 1988;11 (6 pt 2):750-3.

42. Chen M, Lee J, Malvin RL. Central administration of aldosterone increases blood pressure in rats. Clin Exp Hypertens A 1989;11(3):459-72.

43. Gómez-Sánchez EP, Fort CM, Gómez-Sánchez CE. Intracerebroventricular infusion of RU28318 blocks aldosterone-salt hypertension. Am J Physiol 1990; 258(3 pt 1):E482-4

44. Gómez-Sánchez EP, Venkataraman MT, Thwaites D, Fort C. ICV infusion of corticosterone antagonizes ICV-aldosterone hypertension. Am J Physiol 1990;258(4 pt 1):E649-53

45. Geerling JC, Kawata M, Loewy AD. Aldosterone-sensitive neurons in the rat central nervous system. J Comp Neurol 2006;494(3):515-27.

46. Geerling JC, Loewy AD. Sodium depletion activates the aldosteronesensitive neurons in the NTS independently of thrist. Am J Physiol Regul Integr Comp Physiol 2007;292(3):R1338-48.

47. Gomez-Sanchez EP. Dose-response studies of intracerebroventricular infusion of aldosterone in sensitized and non-sensitized rats. J Hypertens 1988;6(6):437-42

48. Peysner K, Henry CA, Malvin RL. Central infusion of aldosterone increases blood pressure by mechanisms independent of Na retention. Clin Exp Hypertens A 1990;12(3):399-414.
49. Matsuguchi H, Sharabi FM, O'Connor G, Mark AL, Schmid PG. Central mechanisms in DOC-salt hypertensive rats. Clin Exp Hypertens A 1982;4(8):1303-21.

50. Takeda K, Nakamura Y, Oguro M, Kawasaki S, Havashi J, Tanabe S, et al. Central attenuation of baroreflex precedes the development of hypertension in DOCA-salt-treated rats. Am J Hypertens 1988; 1 (3 pt 3):23S-25S

51. Takeda K, Nakamura Y, Havashi J, Kawasaki S, Nakata T, Oguro M, et al. Effects of salt and DOCA on hypothalamic and baroreflex control of blood pressure. Clin Exp Hypertens A 1988;10 (suppl 1):289-99.

52. Nakamura Y, Takeda K, Nakata T, Havashi J, Kawasaki S, Lee LC, et al. Central attenuation of aortic baroreceptor reflex in prehypertensive DOCA salt-loaded rats. Hypertension 1988;12(3):259-66.

53. Janiak PC, Lewis SJ, Brody MJ. Role of central mineralocorticoid binding sites in development of hypertension. Am J Physiol 1990;259(5 pt 2):R1025-34.

54. Van den Berg DT, de KloetER, de Jong W. Central effects of mineralocorticoid antagonist RU-28318 on blood pressure of DOCA-salt hypertensive rats. Am J Physiol 1994;267(6 pt 1):E927-33.

55. Gomez-Sanchez EP, Fort C, Thwaites D. Central mineralocorticoid receptor antagonism blocks hypertension in Dahl S/JR rats. Am J Physiol 1992;262(1 pt 1):E96-9.

56. Gómez-Sánchez EP. Mineralocorticoid modulation of central control of blood pressure. Steroids 1995;60(1):69-72.

57. Gomez-Sanchez CE, Zhou MY, Cozza EN, Morita H, Foecking MF, GomezSanchez EP. Aldosterone biosynthesis in the rat brain. Endocrinology 1997:138(8):3369-73

58. Wang W. Chronic administration of aldosterone depresses baroreceptor reflex function in the dog. Hypertension 1994;24(5):571-5.

59. O'Neil RG. Aldosterone regulation of sodium and potassium transport in the cortical collecting duct. Semin Nephrol 1990;10(4):365-74.

60. Vinciguerra M, Mordasini D, Vandewalle A, Feraille E. Hormonal and nonhormonal mechanisms of regulation of the NA,K-pump in collecting duct principal cells. Semin Nephrol 2005;25(5):312-21.

61. Huang BS, Wang H, Leenen FHH. Chronic central infusion of aldosterone leads to sympathetic hyperreactivity and hypertension in Dahl $S$ but not Dahl R rats. Am J Physiol Heart Circ Physiol 2005;288(2):H517-24.

62. Yee KM, Struthers AD. Aldosterone blunts the baroreflex response in man Clin Sci (Lond) 1998:95(6):687-92.

63. Heindl S, Holzschneider J, Hinz A, Sayk F, Fehm HL, Dodt C. Acute effects of aldosterone on the autonomic nervous system and the baroroflex function in healthy humans. J Neuroendocrinol 2006;18(2):115-21.

64. Monahan KD, Leuenberger UA, Ray CA. Aldosterone impairs baroreflex sensitivity in healthy adults. Am J Physiol Heart Circ Physio 2007;292(1):H190-7.

65. Roland BL, Li KX, Funder JW. Hybridization histochemical localizations of 11 beta-hydroxysteroid dehydrogenase type 2 in rat brain. Endocrinology 1995; 136(10):4697-700. 
66. Ahima R, Krozowski Z, Harlam R. Type I corticosteroid receptor-like imunorreactivity in the rat CNS: distribution and regulation by corticosteroids. J Comp Neurol 1991;313(3):522-38.

67. Roland BL, Li KX, Funder JW. Glucocorticoid receptor, mineralocorticoid receptors, 11 beta-hydroxysteroid dehydrogenase- 1 and -2 expression in rat brain and kidney: in situ studies. Mol Cell Endocrinol 1995;111(1):R1-7.

68. Doba N, Reis DJ. Acute fulminating neurogenic hypertension produced by brainstem lesions in the rat. Circ Res 1973;32(5):584-93.

69. Colombari E, Sato MA, Cravo SL, Bergamaschi CT, Campos RR Jr, Lopes OU. Role of medulla oblongata in hypertension. Hypertension 2001;38(3 pt 2):549-54.

70. Arriza JL, Simerly RB, Swanson LW, Evans RM. The neuronal mineralocorticoid receptor as a mediator of glucocorticoid response. Neuron 1988;1(9):887-900.

71. Johnson AK, Loewy AD. Circumventricular organs and their role in visceral functions. In: Loewy AD, Spyer, KM, editors. Central regulation of autonomic functions. New York: Oxford University Press: 1990, cap14, p. 247-67.

72. Pardridge WM, Mietus LJ. Transport of steroid hormones through the rat blood-brain barrier. Primary role of albumin-bound hormone. J Clin Invest 1979;64(1):145-54.

73. Gómez-Sánchez EP. Central hypertensive effects of aldosterone. Front Neuroendocrinol 1997;18(4):440-62.

74. MacKenzie SM, Clark CJ, Fraser R, Gómez-Sánchez CE, Connell JM, Davies E. Expression of 11 beta-hydroxylase and aldosterone synthase genes in the rat brain. J Mol Endocrinol 2000;24(3):321-8.

75. Ye P, Kenyon CJ, MacKenzie SM, Seckl JR, Fraser R, Connell JM et al. Regulation of aldosterone synthase gene expression in the rat adrenal gland and central nervous system by sodium and angiotensina II. Endocrinology 2003;144(8):3321-8.

76. Gomez-Sanchez EP, Samuel J, Vergara G, Ahmad N. Effect of 3betahydroxysteroid dehydrogenase inhibition by trilostane on blood pressure in the Dahl salt-sensitive rat. Am J Physiol Regul Integr Comp Physiol 2005;288(2):R389-93.

77. Talman WT, Perrone MH, Reis DJ. Evidence for L-glutamate as the neurotransmitter of baroreceptor afferent nerve fibers. Science 1980;209(4458):813-5.

78. Loewy AD. Central autonomics pathways. In: Loewy AD, Spyer, KM, editors. Central regulation of autonomic functions. New York: Oxford University Press: 1990, cap6, p. 88-103.
79. Dampney RA. Functional organization of central pathways regulating the cardiovascular system. Physiol Rev 1994;74(2):323-64.

80. Wang T, Edwards GL. Differential effects of dorsomedial medulla lesion size on ingestive behavior in rats. Am J Physiol (Integr Comp Physiol) 1997;273(4 pt 2):R1299-308.

81. Geerling JC, Loewy AD. Aldosterone-sensitive NTS neurons are inhibited by saline ingestion during chronic mineralocorticoid treatment. Brain Res 2006;1115(1):54-64.

82. Sequeira SM, Geerling JC, Loewy AD. Local inputs to aldosteronesensitive neurons of the nucleus tractus solitarius. Neuroscience 2006; 141(4):1995-2005.

83. Geerling JC, Loewy AD. Aldosterone-sensitive neurons in the nucleus of the solitary tract: efferent projections. J Comp Neurol 2006;498(3):223-50.

84. Geerling JC, Loewy AD. Aldosterone-sensitive neurons in the nucleus of solitary tract: Bidirectional connections with the central nucleus of the amygdala. J Comp Neurol 2006;497(4):646-57.

85. Zhang DM, Epstein AN, Schulkin J. Medial region of the amygdala: involvement in adrenal-steroid-induced salt appetite. Brian Res 1993;600(1):20-6.

86. Shekhtman E, Geerling JC, Loewy AD. Aldosterone-sensitive neurons in the nucleus of solitary tract: multisynaptic pathway to the nucleus accumbens. J Comp Neurol 2007;501(2):274-89.

87. Voorhies $A C$, Bernstein IL. Induction and expression of salt appetite: Effects on Fos expression in nucleus accumbens. Behav Brain Res 2006;172(1):90-6.

88. Contreras RJ, Stetson PW. Changes in salt appetite of the area postrema and the nucleus of the solitary tract in rats. Brain Res 1981;211(2):355-66.

89. Hyde TM, Miselis RR. Area postrema and adjacent nucleus of the solitary tract in water and sodium balance. Am J Physiol 1984;247/1 pt 2):R173-182.

90. Curtis KS, Huang W, Sved AF, Verbalis JG, Stricker EM. Impaired osmorregulatory responses in rats with area postrema lesions. Am J Physiol 1999;277(1 pt 2):R209-19.

91. Edwards GL, Belts TG, Power JD, Johnson AK. Rapid-onset "need-free" sodium appetite after lesions of the dorsomedial medulla. Am J Physiol 1993;264(6 pt 2):R1242-7.

92. Geerling JC, Sequeira SM, Loewy AD. Increased number of aldosterone-sensitive NTS neurons in Dahl salt-sensitive rats. Brain Res 2005;1065(1-2):142-6. 